\title{
A Comparative Evaluation of Stochastic-based Inference Methods for Gaussian Process Models
}

\section{Filippone', M. Zhong' ${ }^{2}$ M. Girolami ${ }^{3}$}

\section{Scope of this work}

Gaussian Process models (GPMs) are extensively used in data analysis given their flexible mod作 clly intractable, and therefore it is necessary to resort to approximations. This work focuses on Markov chain Monte Carlo (MCMC) inference techniques. The hierarchical structure of GPM the development of efficient MCMC methods for GPMs. The work employs strategies based on
the effient parame and real data on the basis of convergence speed, sampling efficiency, and computational cost.

\section{Gaussian Process Models - GPMs}

- GPMs:

$$
\underbrace{\text { par }}_{\text {data }} \begin{array}{ll}
p(\text { par }) \\
\text { latent } & p(\text { latent } \mid \text { par })=\operatorname{GP}(\boldsymbol{\mu}(\text { par }), K(\text { data } \mid \text { latent })
\end{array}
$$

- GPM - Probit regression example

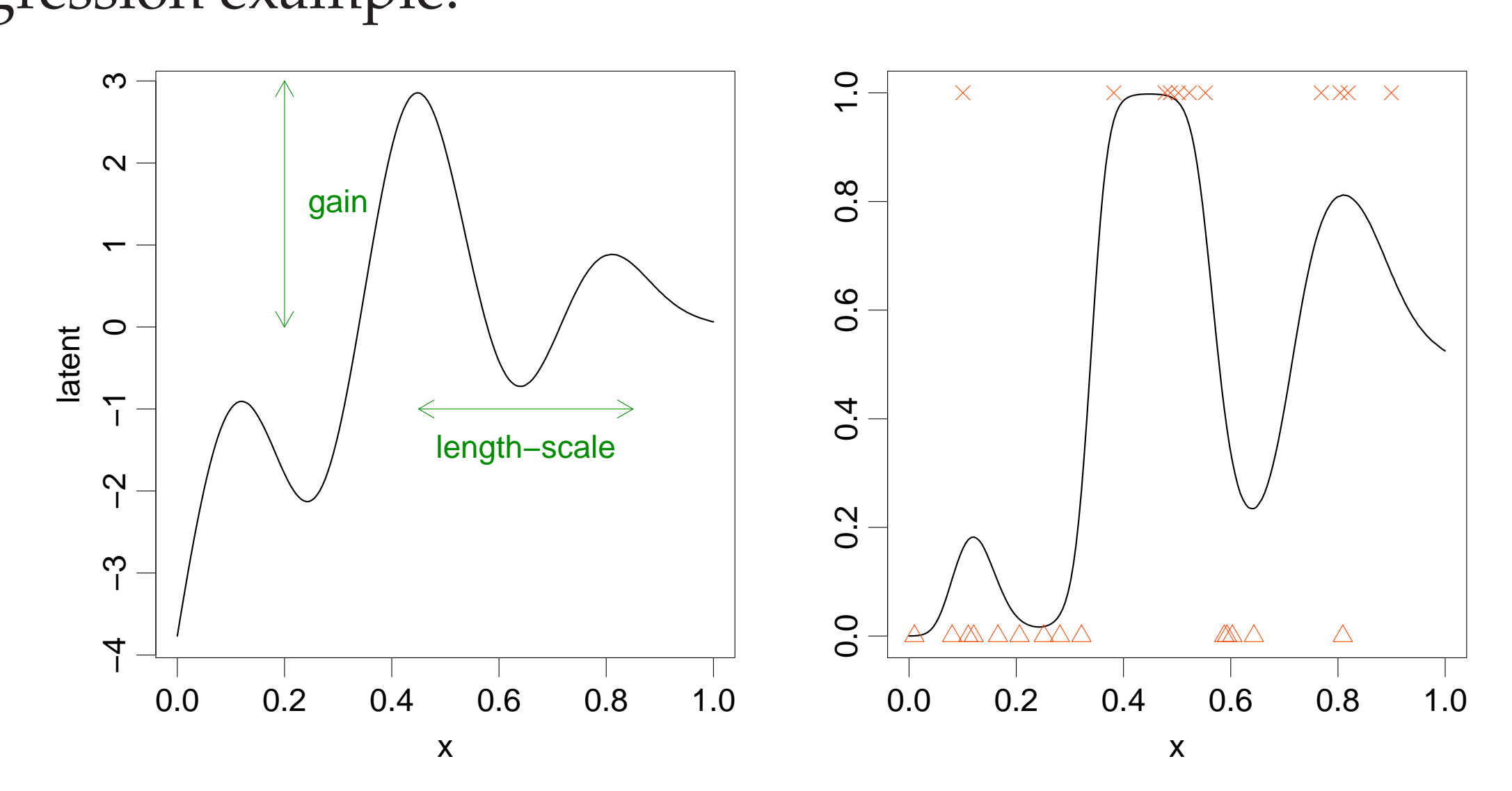

\section{Why MCMC?}

- Comparison MCMC vs Maximum a Posteriori (MAP) using EP

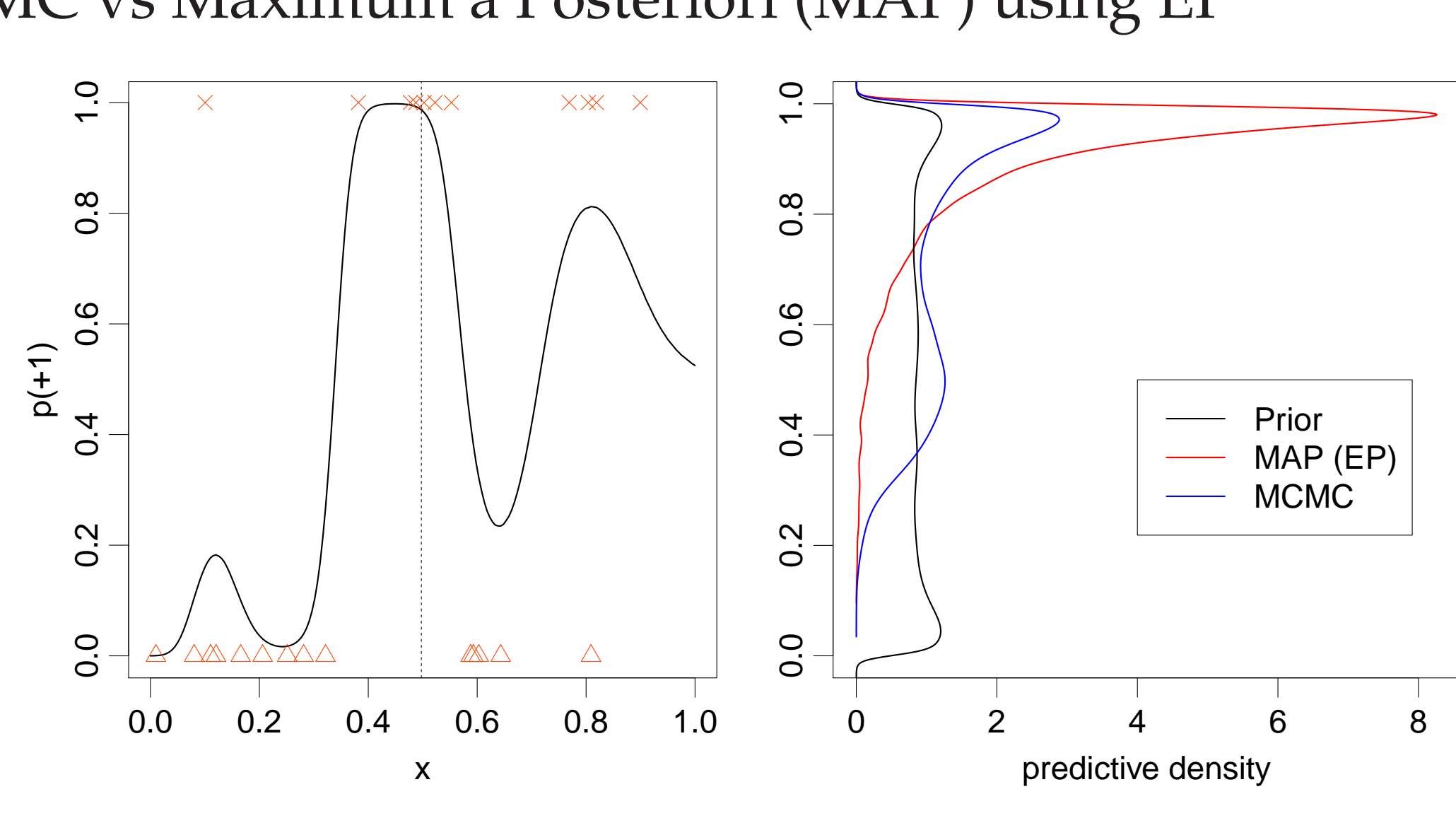

MAP solution does not account for the uncertainty in par! Also:

- Approximate marginal likelihood might be inaccurate

- Quadrature can't be employed if par is large dimensional

\section{References}


.

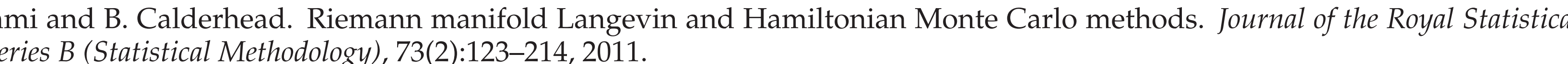

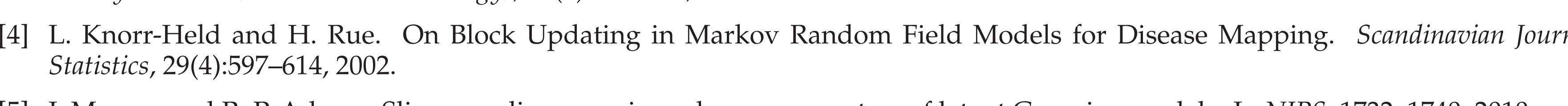


16] I. Murray, R. P. Adams, and D.J.C. Mackay. Elliptical slice sampling. JMLR - Proceedings I Track, 9:541-548, 2010

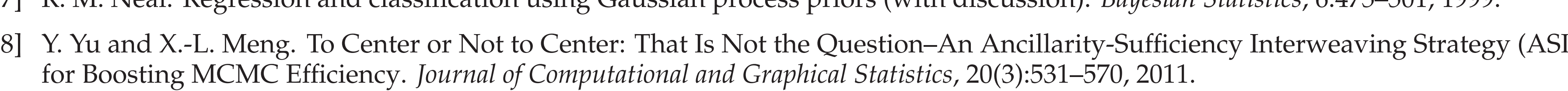

\section{Challenges in employing MCMC for inference in GPMs}

Obvious choice: Sufficient Augmentation (SA) scheme - alternate between:

- Drawing from $p$ (latent|par, data)
$\begin{aligned} & \text { Drawing from } p \text { (par|latent) } \\ & \text { bad idea! }\end{aligned}$

- Need to employ Metropolis within Gibbs steps - waste of computations when rejecting

- Updates of par cost $O\left(n^{3}\right)$

- par can be large dimensional, e.g., Automatic Relevance Determination (ARD) covariance

There are $n$ latent variables (as many as the number of observations)

\section{Mitigating coupling effect through reparameterization}

Ancillary Augmentation (AA) scheme - reparametrization using GP prior covariance $K$ :

$$
\begin{array}{llll}
\qquad K=L L^{\mathrm{T}} & \text { ancillary }=L^{-1} \text { latent } \\
\text { - Replace sampling of par with } \\
p \text { (parlancillary, data) }
\end{array}
$$

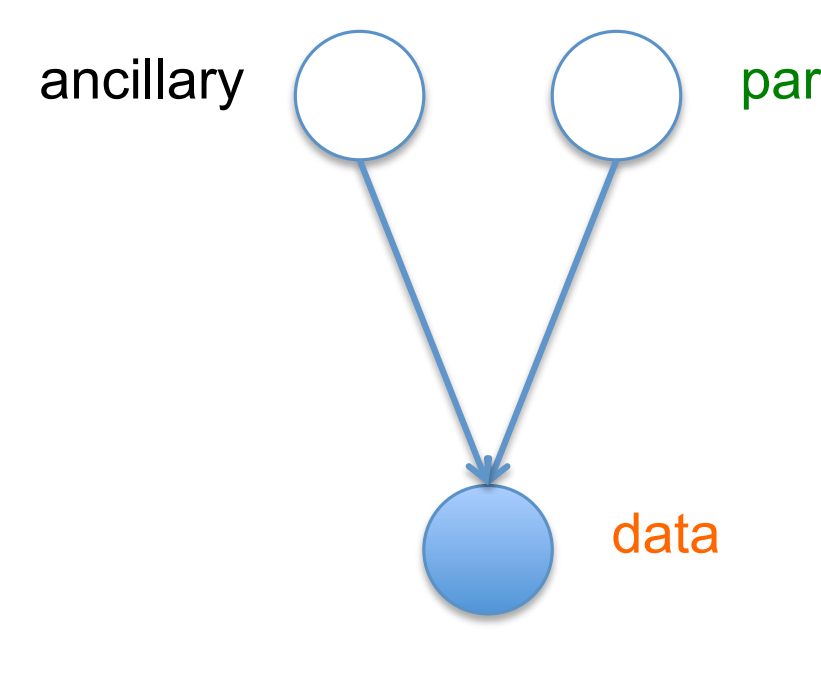

- SURR - Surrogate method in Murray and Adams (2010): reparameterization using cleverly constructed auxiliary variables

KHR - Joint sampler by Knorr-Held and Rue (2002):

propose new par' and latent'|par', data and then jointly Accept/Reject

- ASIS - Interweave AA and SA as in Yu and Meng (2011)

\section{Transition operators for latent variables}

Scaled Metropolis-Hastings (MH) proposals - Neal (1999) - draw $z \sim \mathcal{N}(0, K)$ - MH v1:

- MH v2:

$$
\text { latent }{ }^{\prime}=\text { latent }+\alpha z
$$

$$
\text { latent }^{\prime}=\sqrt{1-\alpha^{2}} \text { latent }+\alpha z
$$

- Scaled Hybrid Monte Carlo (HMC) with mass matrix $M$.

Negative Hessian of the log-posterior is $K^{-1}+\Delta(\mathbf{f})$, with $\Delta$ diagonal.

- HMC v1 - Christensen et al. (2005)

$$
M=K^{-1}+\Delta(\mathbf{0})
$$

- HMC v2 - proposed in this work

$M=K^{-1}$

Both are conveniently implemented by deriving HMC specifying $M^{-1}$

- Manifold MCMC - Girolami and Calderhead (2011)

Simplified Manifold MALA (SMMALA) gradient and curvature information

Elliptical Slice Sampling (ELL-SS) (Murray et al. 2010) is an adaptation of slice sampling to sample latent variables in GPMs
Transition operators for parameters

Metropolis-Hastings (MH) random walk

Hybrid Monte Carlo (HMC) gradient information

Simplified Manifold MALA (SMMALA) gradient and curvature information

\begin{tabular}{|c|c|c|c|c|c|}
\hline & \multicolumn{3}{|c|}{$n=400$} & & \\
\hline & $d=2$ & & $d=10$ & & 2 \\
\hline & $2124(12$ & 而 & $77(33)$ & 血 & \\
\hline $\begin{array}{l}\text { HMC } \\
\text { SMMALA }\end{array}$ & $12556(661)$ & In & $293(137)$ & 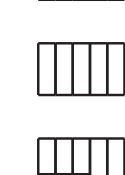 & $\begin{array}{l}T(\bar{\lambda}+1) \\
T(d+p)\end{array}$ \\
\hline
\end{tabular}

\section{Results}

- Models employ a radial basis function ARD covariance

- Convergence speed measured using $\hat{R}$ statistics. To visualize convergence between 1000 and 20000 iteration

$$
0<1.1<\text { : }<1.3<\boldsymbol{l}<2<\boldsymbol{I}
$$

Efficiency measured through (the minimum across variables) Effective Sample Size (ESS)

Table 1: Comparison of transition ope



Table
steps.

Table 3: AA
sion GPMS.



Table 4: Comparison of different strategies to sample latent, parldata in four UCI data sets modeled using logiss



\section{Conclusions}

Sampling from the posterior of latent variables can be efficiently done in a number of ways

AA scheme with par sampled using the MH algorithm seems a good compromise between efficiency and cost

Sampling efficiency is sometimes less than $1 \%$ for the best sampling strateg

- Fully automated MCMC for GPMs still an open problem 